\title{
Telmatophilus Heer, 1841 (Coleoptera: Cryptophagidae) of western Palaearctic region
}

\author{
José Carlos Otero
}

Otero, J. C. 2012: Telmatophilus Heer, 1841 (Coleoptera: Cryptophagidae) of western Palaearctic region. - Entomol. Fennica 23: 113-120.

Three species of Telmatophilus Heer, 1841 in the Western Palaearctic Region are revised. Basing on studies of the types of the species, Telmatophilus brevicollis Aubé, 1862, T. caricis (Olivier, 1790) and T. typhae (Fallén, 1802) are redescribed. Two new synonymies are established: Cryptophagus sparganii Ahrens, 1812 = Telmatophilus caricis (Olivier, 1790) and Cryptophagus schonherrii Gyllenhal, $1808=$ Telmatophilus typhae (Fallén, 1802). Species are redefined according to their morphology, including that of the genitalia. A key of the studied species is included.

J. C. Otero, Departamento de Zoología y Antropología Física, Facultad de Biología, 15782 Santiago de Compostela, Spain; E-mail: josecarlos.otero @usc.es

Received 31 May 2011, accepted 21 July 2011

\section{Introduction}

As a result of the variability of certain morphological features (shape and size of the pronotum, colour, size, and so on) there have always existed differences of opinion in the classification of species in the Telmatophilus genus. This way, Crotch (1865) takes into account five species in the British Isles: T. sparganii (Ahrens, 1812); T. caricis (Olivier, 1790); T. brevicollis Aubé, 1862; T. typhae Fallén, 1802) and T. schonherrii (Gyllenhal, 1808). In his monograph about the European species, Reitter (1875) added four new species to those previously known: T. longicollis Reitter, 1875; T. integricollis Reitter, 1875; T. rufus Reitter, 1875 and T. pumilus Reitter, 1875. All the subsequent authors started from this point (Falcoz 1929, Portevin 1931, etc.). Karaman (1961) studied the collection of the Natural History Museum in Sarajevo, and considering the anatomy of the female and male genitalia, she reduced the number of species to four: $T$. caricis, $T$. brevicollis, T. typhae and T. balcanicus Karaman, 1961. Recently Sasaji (1978) described one new species from Japan, and Johnson et al. (2007) point out five species for the Western Palaearctic Region.

I have studied the type specimens of different species, besides the material coming from different European localities. I observed that the only differences appear in the colour of the elytra and the legs and in the size of the pronotum. I could not find any difference in the morphology of the genitalia or in the metatibiae. Consequently, I propose the exposed synonymies in each case and reduce the species present in the western Palaearctic Region to three.

\section{Material and methods}

Terminology and measurements for the new species follow Otero $(1997,2001)$. Structures were measured under an Olympus SZX12 stereo- 
microscope equipped with an image analysis system (MicroImage Version 4.0 for Windows).

The following acronyms are used throughout the paper to refer to the collections, in which the examined specimens are deposited:

CA: coll. F. Angelini, Francavilla Fontana, Italy CB: coll. Bratton, Wales, United Kingdom

MB: Museo Barcelona, Barcelona, Spain

MBA: Museum für Naturkunde der A. Humboldt-Universität, Berlin, Germany

MBH: Hungarian Museum of Natural History, Budapest, Hungary

ME: Museum für Naturkunde, Erfurt, Germany

MG: Museo Civico di Storia Naturale G. Doria, Genova, Italy

NHMW: Naturhistorisches Museum, Wien, Austria

MHML: Muséum D'Histoire Naturelle de Lyon, Lyon, France

MLUHW: Martin-Luther-Universität HalleWittenberg, Germany

MNCN: Museo Nacional Ciencias Naturales, Madrid, Spain

\section{Taxonomy}

\subsection{Telmatophilus brevicollis Aubé, 1862} Figs. 1a-g

Telmatophilus brevicollis Aubé, 1862. Ann. Soc. Entomol. Fr., (4), 2: 72

Telmatophilus rufus Reitter, 1875. Dtsch. Entomol. Z., 19(3): 226

Telmatophilus parens Rey, 1889. Échange, 5: 36 Telmatophilus balcanicus Karaman, 1960. Verh. XI Int. Kongr. Entomol., Wien, 1: 74

Material examined. 3 Syntypes (without locality or date) labeled T. parens (coll. Rey) (MHNL). Austria: Gars, 4 ex; Grunnstadt, 3 ex; Neusield, 3 ex; Neusiedlersee, Bei Podersdorf, VI.1930, 8 ex (NHMW). Germany: Leipzig, Zöbigker and Fenstern, 15.VI.1929, 1 m. (MLUHW). Greece: Ahaia, Kalogria, 1-2.V.2004, 2 ex (leg. F. Angelini) (CA). Hungary: Hungar bor, 2 ex (NHMW); Békés, Sarkad, 25.V.1982, 7ex; Gyor M-S m, Tarnokrét, 18.VI.1997, 2 ex; Nógrád m,
Degrelypalank, 25.V.1993, 3 ex; Pest m., Szigetbecse, 30.IV.1995, 1 ex (MBH). Italy: Basilicata, Lago Pantano di Pignola, 7.XI.1993, 8 ex; Basilicata, Ris. WWF La Pignola, 11.VII.1992, 13 ex; 25.VI.1993, 15 ex; 28.IV.1994, 3 ex; 23.IV.2000, 2ex; Pollino, 17.V.1995, 1 ex (leg. F. Angelini) (CA); Emilia, Brugueto, 15.VI.1918, 6 ex (MG); Toscana, Casini Serana, La Querciola, 3.VI.2002, 1 ex; Toscana, Padule Fucchio, 4.V. 2002, 1 ex; Toscana, Sesto Fiorentino, La Querciola, 10.V.2002, 2 ex (leg. S. Rocchi) (MG). Spain: Mallorca, Artá, V.1927, 3 ex (MB).

Redescription. Length 2.3-2.6 mm. Body moderately convex with dense and thin punctation. Colour of body dark brown, and antennae and legs reddish-brown. Greyish pubescence. Metathoracic wings developed. Antennae (Fig. 1c) robust, almost reaching back edge of pronotum. Pronotum (Figs. 1a, b) very convex, wider than long but narrower in front part and with greatest width behind middle; lateral margins of pronotum finely crenulated and base provided with two small pits linked by a transverse groove. Last abdominal sternite of males foveolate in middle and surrounded by a short pubescence. Distal part of aedeagus (Fig. 1d) with two tips curved towards middle; central body elongate and internal sac provided with small teeth. Parameres (Fig. 1e) short, as long as wide, and two setae of different length in apical region. Female hemisternite presented in Fig. 1f. Styles (Fig. 1f) similar to those of T. typhae, but thinner. Spermateca illustrated in Fig. 1g.

Geographic distribution. Present in the majority of Southern and Central European countries (Johnson et al. 2007).

Biology. The larva depends on the seeds of Sparganium erectum L. (Perris 1877, Bratton 2000).

\subsection{Telmatophilus caricis (Olivier, 1790)}

Figs. 2a-g

Ips caricis Olivier, 1790. Entomologie, 2(18): 15

Dermestes obscurus Fabricius, 1792. Entomol. Syst., 1(1): 233

Bostrichus obscurus (Fabricius): Thunberg, 1798. Diss. Acad. Mus. Nat. Acad. Ups., Append. 6: 114 
Fig. 1. Telmatophilus brevicollis Aubé. - a. General view, male $(A=0.400 \mathrm{~mm})$. - b. Pronotum $(A=0.363 \mathrm{~mm})$. -c. Antenna $(A=0.400$ $\mathrm{mm})$. - d. Dorsal view aedeagus $(A=0.190 \mathrm{~mm})$. -e. Paramere $(A=0.190$ $\mathrm{mm})$. - g. Spermatheca. $-\mathrm{f}$. Hemisternite and stylus $(A=0.270 \mathrm{~mm})$.
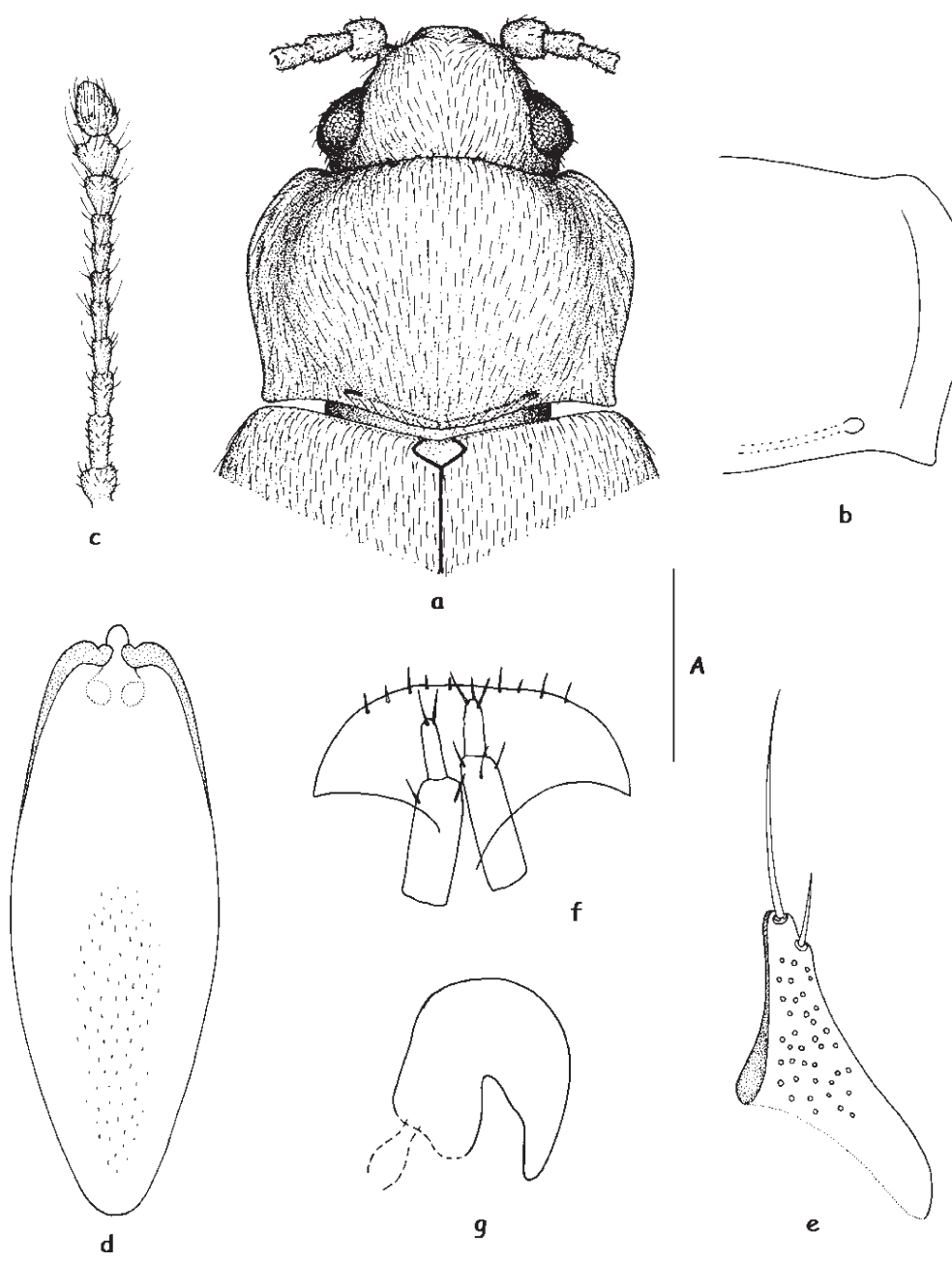

Byturus caricis (Olivier): Latreille, 1804. Hist. Nat. Gén. Part. Crustac. Insectes, 10: 42 Cryptophagus caricis (Olivier): Gyllenhal, 1810. Insecta Suec. Descr., 2: Add. ad part. I, p. iv Cryptophagus sparganii Ahrens, 1812. Neue Schr. Nat.forsch. Ges. Halle, 2(2): 21; syn. n. Telmatophilus caricis (Olivier): Heer, 1841. Fauna Coleopteror. Helv., 1(3): 417

Telmatophilus sparganii (Ahrens): Sturm, 1841. En: Heer, Fauna Coleopteror. Helv., 1(3): 417 Limnesius caricis (Olivier): Sturm, 1843. Cat. Käfer-Samml.: 93

Limnesius sparganii (Ahrens): Sturm, 1843. Cat. Käfer-Samml.: 93

Telmatophilus longicollis Reitter, 1875. Dtsch. Entomol. Z., 19(3): 225

Telmatophilus substriola Rey, 1889. Échange, 5: 36
Material examined. 2 Syntypes (MLUHW) (labeled T. substriola) (MHNL). Austria: Neusiedlersee, Bei Podersdorf, VI.1930, 5 ex; Sartorius, 1876, 4 ex (as T. sparganii); Gars, 5 ex; Neusiedlersee, Bei Podersdorf, VI.1929, 7 ex; Paskau, 5 ex; Tirol, 4 ex; Wien, Umgebung Haberditz, 1 ex; (MNMW). Bosnia and Herzegovina: Dervente, 2 ex; Caplijina; 1 ex (leg. D. Grabowski) (MBH). Croatia: Dalmatia, 2 ex (leg. E. Reitter); 1 ex (leg. E. Reitter) (MBH). Hungary: Balatonlelle, 1 ex; Békés m. Sarkad, 25.V.1982, 12 ex; Bodrogszegi, Szgi v. áll, 12.VI.1997, 1 ex; Karcsa, Becsked, 25.V.1994, 1 ex; Budapest, 26.IV.1913, 1 ex; 9.VI.1915, 1 ex; Kiskunsági, 21.II.1979, 1x; 14.VI.1979, 1 ex; 10.IX.1979, 1 ex; 13.XI.1979, 1 ex (leg. Adám); Pest m. Pécel, 25.V.2006, 1 ex; Satoraljaújhely, Gyalmos, 23.X.1993, 1 ex; Gyor, 14.VII.1941, 1 

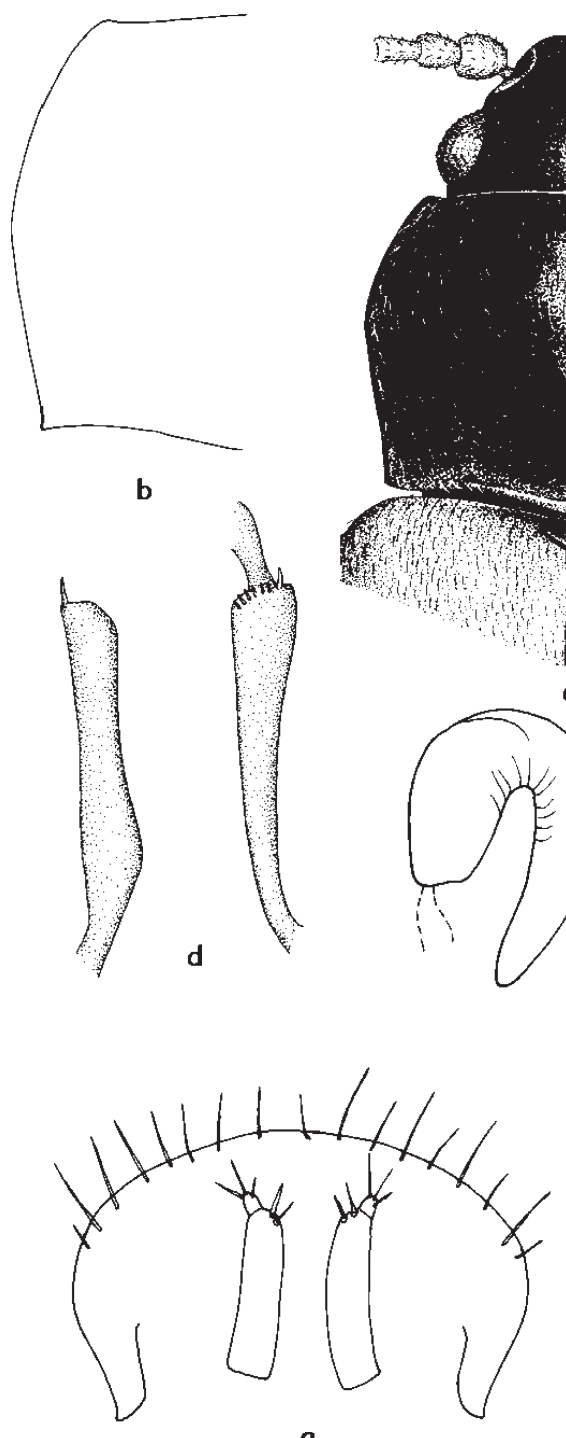

9

ex (leg. Révy) Satoraljaújhely, Paup-to, 28.VII.1994, 1 ex; Satoraljaújhely, Bibérc, 16.V. 1997, 1 ex (coll. Hgyssy) (MBH); Tiszassüñy, 19.V.1956, 1 ex (leg. Z. Kaszab); Zalavár, 8.VI.1913, 1 ex (leg. Z. Kaszab) (MBH). Italy: Basilicata, Lago di Pignola, 7.XI.1993, 2 ex; Basilicata, Pignola, Ris WWF L. Pignola, 11.VII.1992, 22 ex; 27.X.1995, 20 ex; 2.IV.2000, 2 ex (leg. F. Angelini) (CA); Sardegna, Estagno Ispiritu Santo, V.1974, 1 ex (leg. M. Franciscolo); Toscana, Bientina, 7.VII.1997, 3 ex; Toscana, L. Sibolla, 16.V.1995, 1 ex; 23.IV.1996, 1 ex; Toscana, La Querciola, Caserana, 27.IV.2001, 3 ex; 3.VII.2002, 2 ex; Toscana, La Querciola,
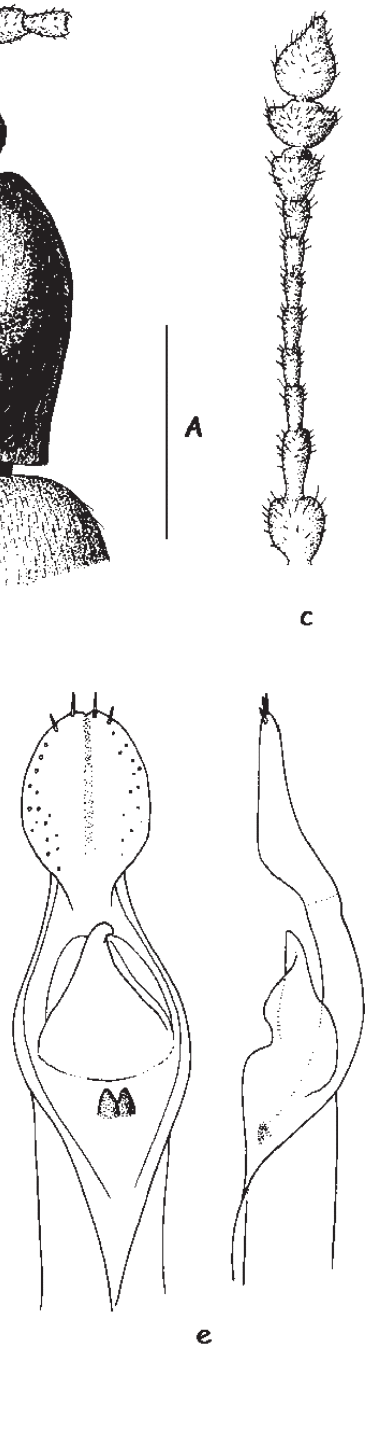

Fig. 2. Telmatophilus caricis Olivier. - a. General view, male $(A=$ $0.560 \mathrm{~mm})$. - b. Pronotum $(A=0.400 \mathrm{~mm})$. c. Antenna $(A=0.330$ $\mathrm{mm})$. - d. Hind tibia male and female $(A=$ $0.340 \mathrm{~mm})$. - e. Dorsal view and lateral aedeagus $(A=0.350 \mathrm{~mm})$. $-\mathrm{f}$. Spermatheca. $-\mathrm{g}$. Hemisternite and stylus $(A=0.200 \mathrm{~mm})$.

Sesto Fiorentino, 18.IV.2002, 2 ex; Toscana, Lago di Porta, 14.IV.1997, 2 ex; 3.IV.2001, 1 ex; Toscana, Padule de Fucecchio, 15.IV.1996, 8 ex; 4.V.2000, 4 ex; Toscana, Stagno di Focognano, Campi di Bisenzico, 5.V.1996, 1 ex; 7.IV.1997, 6 ex; 12.IV.1997, 6 ex (leg. S. Rocchi). Moldavia: Bessarabia, 1983, 2 ex (MBH). Rumania: Tulcea, 26.VII.1959, 1 ex (leg. Gozmány) (MBH). Slovakia: Jurici, 9-20.VI.1915, 1 ex (leg. J. Fodor); Kassa, 1 ex (leg. Horváth.) (MBH).

Redescription. Length 2.4-2.8 mm. Body oblong, convex, dense and finely punctate, covered with fine greyish-white, short $(\mathrm{L}=41.2-44.2 \mu \mathrm{m})$ and horizontal pubescence. General colour black 
with elytra brown or reddish brown and antennae and legs ferruginous; sometimes body yellowish brown and elytral suture blackish, same as another line, with same colour, situated in external margin of elytra. Metathoracic wings developed. Eyes $(\mathrm{L}=0.139 \mathrm{~mm})$ very convex and prominent, with facets of $18 \mu \mathrm{m}$ diameter. Antennae (Fig. 2c) not reaching back edge of pronotum; second antennomere $1.43 \times$ as long as third one. Pronotum (Figs. 2a, b) slightly wider than long $(\mathrm{RD}=$ 1.15), slightly narrower in front part and, usually, slightly sinuous in front of posterior angles. Sides finely crenulate. Punctation dense; distance between puncture lower than their diameter $(\varnothing=$ 8.6-9.5 $\mu \mathrm{m}$ ). Elytra with a fine sutural groove and punctation less dense and as fine as in pronotum: distance between puncture of $1.5 \times$ diameter $(\varnothing=$ 20-22 $\mu \mathrm{m}$ ). In males metatibiae (Fig. 2d) dilated in basal third and last abdominal sternite foveolate. Aedeagus presented in Fig. 2e. Parameres with half-moon shape, with a distal seta and some smaller ones in edges. Internal sac with two triangular spines. Females have a rounded hemisternite (Fig. 2g) with many apical setae; styles (Fig. $2 \mathrm{~g}$ ) small, oval and with some small setae in distal position; spermateca presented in Fig. $2 \mathrm{f}$.

Geographic distribution. Present in the majority of countries in the Western Palaearctic Region (Johnson et al. 2007).

Biology. Lives on Typha sp. and Sparganium sp. The larva grows in its seeds during June and July (Bratton 1997).

\subsection{Telmatophilus typhae (Fallén, 1802) Figs. 3a-i}

Cryptophagus typhae Fallén, 1802. Obs. Entomol., 1: 16

Cryptophagus schonherrii Gyllenhal, 1808. Insecta Suec. Descr., 1: 173; syn. n.

Telmatophilus typhae (Fallén): Heer, 1841. Fauna Coleopteror. Helv., 1(3): 417

Limnesius schoenherrii (Gyllenhal): Sturm, 1843. Cat. Käfer-Samml.: 93

Limnesius typhae (Fallén): Sturm, 1843. Cat. Käfer-Samml.: 93

Telmatophilus pumilus Reitter, 1875. Dtsch. Entomol. Z., 19(3): 226

Telmatophilus prothetelie Kemmer, 1914. Entomol. Tidskr., 35: 87
Material examined. 2 Syntypes (labeled $T$. schoenherri) (MLUHW). Austria: Gars, 8 ex; Sartorius, 1876, 9 ex; Wien, 1921, 3 ex (as T. schoenherri) (NHMW). Bulgaria: Sandanski, 25.VI.1984, 1 ex (coll. Uhlig) (MBA). Caucasia: 1 ex (Leg. E. Reitter). Denmark: E-Jutland, Glatved, 5.VII.1991, 1 ex (leg. Munk). England: Penychain, 28.VII. 2000, 10 ex (as T. schoenherrii) (leg. J. Bratton) (CB). France: Argentan, 13.VII.1934, 2 ex (MB); Loira, Candes St Martin, 19.VIII.1991, 1 ex (leg. F. Montemurro). Germany: Sachsen, Frohburg, 21.V.1972, 2 ex; Hellsee, Lanke, 10.I.1988, 2 ex (MBA). Greece: Ahaia, Kalogira, 1-2.V.2004, 1 ex (leg. F. Angelini) (CA). Hungary: BácsKiskum m., 29.V.1983, 1 ex (leg. Adám); Baranya m., Totszentgyörgy, 25.VI.2006, 1 ex (O. Merkl); Békés m. Kategyháza, 7.V.1982, 2 ex; Békés, Sarkad, 25.V.1982, 1 ex; Bukki N.P., Bélapátfalva, 14.VI.1984, 1 ex (leg. O. Merkl); Bursod m. Rakaka, 6.VI.1997, 1 ex; Csongrad m. Szentes, 28.VII.1998, 1 ex; Gyor M-S m, Rajka, Dona-part, 28.VI.2001, 1 ex; Hortobàgy N.P., Egyek, 5.VIII.1975, 3 ex (leg. L. Papp); Hortobàgy N.P., Tiszacsege, 2-5.VI.1975, 2 ex (leg. L. Papp); Heves meggye, Kerecsend, Fácános, 12.VI.2010, 3 ex (leg. O. Merkl); Kelebia, Hastavak, 8.V.1962, 1 ex (leg. Endrody); Kiskunsági N. P., Lakitelek, 21.II. 1979, 1 ex (leg. Adám); Mariabesnyo, 12.VI. 1927, 1 ex (leg. J. Fodor); Somogy m. 24.V.1995, 1 ex; Somogy m. Fonyod, 12.VI. 1985, 1 ex; Somogy m. Gyekenyes, 7.VI.1993, 2 ex; Veszprem m. 27.VII.2002, 2 ex. (MBH). Italy: Abruzzo, Carsoli, 30.V.1993, 5 ex (leg. G. Pace); Basilicata, Pantano di Pignolo, 25.VI.1992, 1 ex; Policoro, 20.VI.1996, 1 ex ; 17.IX.2000, 2 ex (leg. F. Angelini) (CA); Bagnaria, 13.VI.1989, 6 ex (leg. G. Pace); Lazio, Ceri, 25.VIII.1972, 8 ex, (MG); Lombardia, Brivio, 10.V.1934, 1 ex (MG); Carsoli, 30.V.1993, 5 ex; Godiasco, 25.XI.1990, 1 ex; Lombardia, Mantova, 26.IV.1971, 1 ex (leg. Scaglioni); 7.V.1988, 3 ex (leg. P. Cornacchia); Puglia, Daunia, Celenza Valfort, 15.V.2001, 5 ex; Sardegna, Lago Baratz, 23.V.1995, 3 ex; Toscana, Bientina, 24.VI.1996, 3 ex; 12.V.1997, 1 ex; Toscana, Ferrone, 14.VIII.1998, 1 ex; Toscana, Lago di Porta, 15.IX.2001, 1 ex; Toscana, Orgia, 15.V.1997, 2 ex (leg. A. Bordoni); Rivanazzano, 8.IV.1990, 8 ex (leg. G. Pace); 

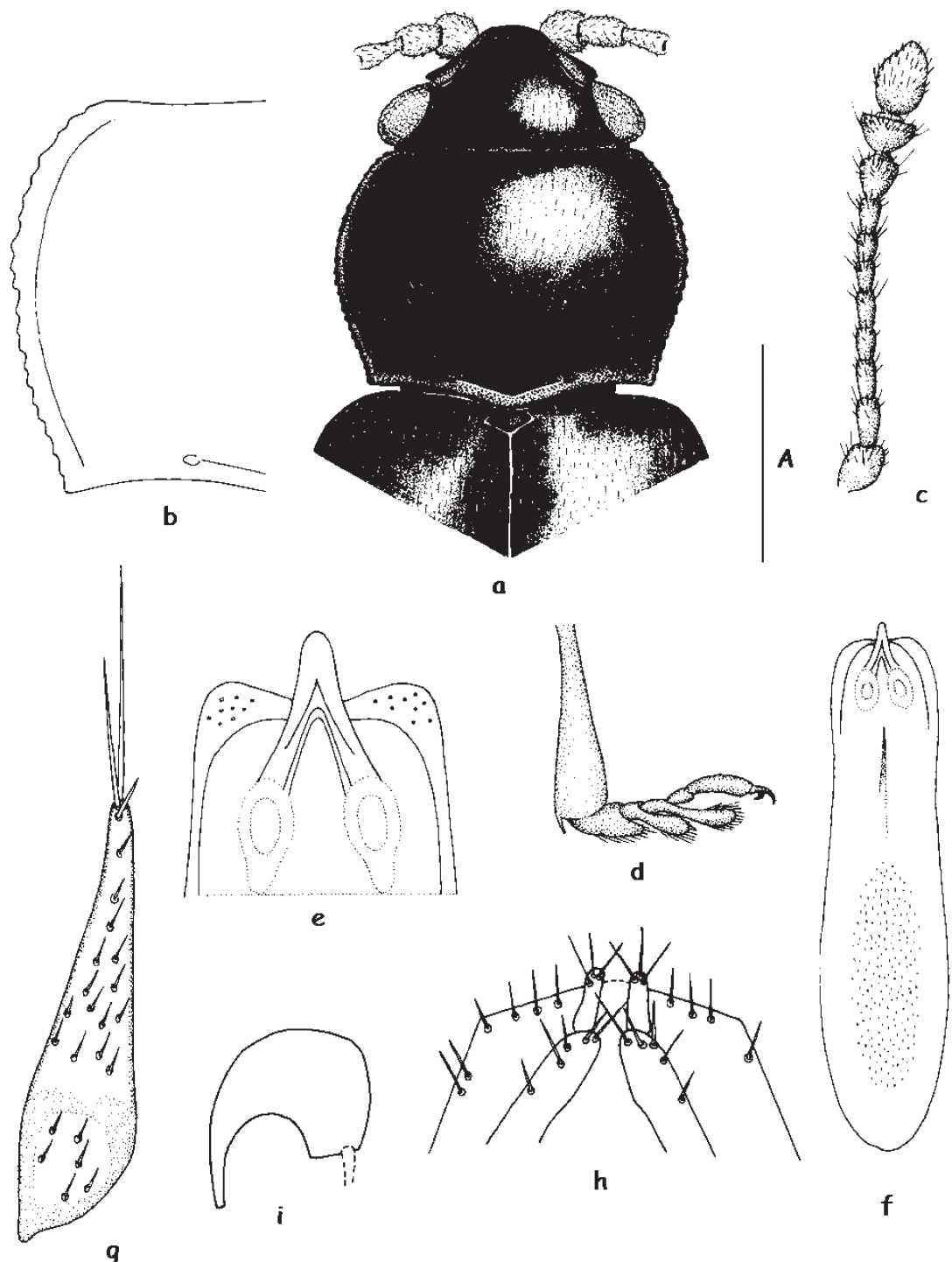

Fig. 3. Telmatophilus typhae Fallen. - a. General view, male $(A=0.355 \mathrm{~mm}) .-b$. Pronotum $(A=0.288$ $\mathrm{mm})$. - c. Antenna ( $\mathrm{A}=$ $0.250 \mathrm{~mm})$. - d. Front tarsus of female $(A=$ $0.342 \mathrm{~mm}$ ). - e, f. Dorsal view aedeagus $(A=0.184 \mathrm{~mm})$. - g. Paramere $(A=0.184$ $\mathrm{mm})$. - i. Spermatheca. - h. Hemisternite and stylus $(A=0.266$ $\mathrm{mm}$ ).

Puglia, F. Lato, 2.I.1977, 1 ex (leg. F. Angelini) (CA). Kazakhstan: Almaty, 22 km N. Masak, 21.VI.1996, 2 ex (MBH). Russia: Moskau, Tschaschnikow, 21.VI.1959, 1 ex; Swnnigorad, 15.VI.1958, 2 ex; 9.VIII. 1958, 2 ex (MBA). Slovakia: Ajdousaina, Rasa Vipava, 17.VI.1996, 1 ex (leg. S. Zoia). Spain: Barcelona; Vallvidrera,.VI.1937, 4 ex (MB); Cádiz, Laguna Torage, 6.VI. 1977, 2 ex (leg. R. Outerelo); Gerona, Sal, 14.IX.1979, 2 ex (leg. J. de Ferrer); La Coruña, Villa Rutis, VIII.1908, 2 ex (MNCN); Madrid, 2 ex; El Escorial, 1 ex (MNCN); Mallorca, Cappdepera, VI.1930, 8 ex (MB); Valencia, Sagunto, 4 ex (MB). Turkey: Beysehir,
Umg. Beysehir Gölü, 6.VIII. 1990, 1 ex (leg. A. Weigel) (ME); Samsun prov., Lâdik, Lâdik Gölü, 7.VI.2009, 1 ex (leg. F. Angelini) (CA); Turkestan, 12 ex (NHMW).

Redescription. Length 1.5-2.3 mm. Body oblong, convex, dense and finely punctate, covered with whitish, short and horizontal pubescence. Colour bright black with antennae and legs ferruginous or yellowish; sometimes femora, distal extreme of tibiae and antennomeres first, ninth and tenth black. Metathoracic wings developed. Big eyes $(\mathrm{L}=0.140 \mathrm{~mm})$, convex and with facets of 10-12 $\mu \mathrm{m}$ diameter. Antennae (Fig. 3c) short, not reaching back edge of pronotum, with second 
antennomere $1 / 5$ longer than the third one. Pronotum (Figs. 3a, b) as narrowed in front as in back, regularly rounded and with margins finely crenulate; a line on each side, more or less visible, parallel to lateral edge. Base with two small pits linked by a transverse groove. Punctation strong and dense, distance between punctures equal to diameter $(\varnothing=10 \mu \mathrm{m})$. Elytra with a suture thin and different. Punctation fine and dispersed, distance between punctures higher to diameter $(\varnothing=6$ $\mu \mathrm{m})$. Aedeagus (Figs. 3e, f) wide, rounded in distal region and slightly shortened in its central edge; central body distally elongate and digitiform. Internal sac with many tiny spines. Parameres (Fig. 3g) long and narrow with two long apical setae. In females protibiae (Fig. 3d) slightly dilated in ends, hemisternite (Fig. 3h) with distal edges straight and provided with many setae, styles (Fig. 3h) $4 \times$ as long as wide and present three or four terminal setae, spermateca presented in Fig. 3i.

Geographical distribution. Widely spread in Europe (Johnson et al. 2007) and North America (Hoebeke \& Wheeler 2000).

Biology. Lives on Typha latifolia L. and other aquatic plants feeding on pollen (Falcoz 1929, Bratton 2000).

\subsection{Key of species}

1. Body slightly convex. Pronotum $1.4 \times$ as wide as long

- Body very convex. Colour brownish-black, unicoloured; legs and antennae reddish. Punctation dense and fine; short pubescence. Pronotum as long as wide. Male metatibiae dilated in basal third (Fig. 2d). Aedeagus presented in Fig. 2e. Parameres with half-moon shape, with a distal seta and some smaller ones in edges. Internal sac with 2 triangular spines. Females have a rounded hemisternite (Fig. 2g) with many apical setae; styles (Fig. $2 \mathrm{~g}$ ) small, oval and with some small setae in distal position; spermateca presented in Fig. 2f. Length 2.4-2.8 mm T. caricis (Olivier)

2. Colour black or brownish ferruginous. Pronotum almost $2 \times$ as wide as long, narrower in front part (Figs. 1a, b). Distal part of aedeagus (Fig. 1d) with 2 tips curved towards middle; central body elongate and internal sac provided with small teeth. Parameres (Fig. 1e) short, as long as wide and, 2 setae of different length situated in apical region. Hemisternite of females presented in Fig. 1f. Styles (Fig. 1f) similar to those of $T$. typhae, but slightly thinner. Spermateca shown in Fig. $1 \mathrm{~g}$. Length: 2.3-2.6 mm. T. brevicollis Aubé - Colour bright black, antennae and legs ferruginous. Covered with a short, whitish and horizontal pubescence. Pronotum $1.5 \times$ as wide as long, parallel-sided. Aedeagus (Figs. $3 \mathrm{e}, \mathrm{f})$ wide, rounded in distal region and slightly shortened in its central edge; central body distally elongate and digitiform. Internal sac with many tiny spines. Parameres (Fig. 3g) long and narrow with 2 long apical setae. In females protibiae (Fig. 3d) slightly dilated in ends, hemisternite (Fig. 3h) has distal edges straight and with many setae, styles (Fig. 3h) $4 \times$ as long as wide and with 3 or 4 terminal setae, spermateca presented in Fig. 3i. Length $1.5-2.3 \mathrm{~mm} \quad$ T. typhae (Fallén)

Acknowledgements. I would like to express my acknowledgement to colleagues and Institutions that allowed me to study specimens of the studied genus.

\section{References}

Bratton, J. 1997: Notes on the distribution and hostplants of Telmatophilus species (Cryptophagidae). - Latissimus 8: 7-9.

Bratton, J. 2000: More on Telmatophilus schoenherri and T. brevicollis (Cryptophagidae). — Latissimus 4.

Crotch, G. R. 1865: Notes onte genus Telmatophilus, with description of a new British species. - The Entomologist 2: 209-210.

Falcoz, L. 1929: Tableaux analytiques des Coléoptères de la faune Franco-Rhénane. Famille XXXIII Cryptophagidae. - Miscellanea Entomologica 31-32: 1-48.

Hoebeke, E. R. \& Wheeler, A. G. 2000: Telmatophilus typhae (Fallén) (Coleoptera: Cryptophagidae), a Palearctic cattail, specialist established in the Canadian maritime provinces. - Proceedings of the Entomological Society of Washington 102: 398-402.

Johnson, C., Otero, J. C. \& Leschen, R. A. B. 2007: Cryptophagidae. - In: Löbl, I. \& Smetana, A. (eds.), Catalogue of Palaearctic Coleoptera, Vol. 4. Elateroidea, Derodontoidea, Bostrichoidea, Lymexyloidea, Cleroidea, Cucujoidea. Apollo Books. Stenstrup: 513-531.

Karaman, Z. 1961: Kopulatiosapparat des Genus Telmatophilus (Cryptophagidae, Col.). — Verhandlungen 
des XI Internationaler Kongress für Entomologie Wien 1: 71-74.

Otero, J. C. 1997: Three new species and distributional records of Micrambe C. G. Thomson, 1863 and Cryptophagus Herbst, 1792 (Coleoptera: Cryptophagidae) from Israel and Turkey. - Revue Suisse de Zoologie 104: 207-216.

Otero, J. C. 2001: Records of Cryptophagus Herbst, 1792 from the Himalaya, with a new species from Thailand (Coleoptera: Cryptophagidae). - Revue Suisse de Zoologie 108: 987-992.
Perris, M. E. 1877: Larves des Coléoptères. - Annales de la Société Linnéenne de Lyon: 590 pp.

Portevin, G. 1931: Histoire Naturelle de Coléoptères de France. 2. Edit. - Paul Lechevalier, Paris, 502 pp.

Reitter, E. 1875: Die bekannten Telmatophilus-Arten, übersichtlich dargestellt. — Deutsche Entomologische Zeitschrift 19: 225-228.

Sasaji, H. 1987: Contribution to the taxonomy of the superfamily Cucujoidea (Coleoptera) of Japan and her adjacent districts, III. - Memoirs Faculty Education, Fukui University (Ser. II) Natural Science 37: 23-55. 